\title{
El CESID: Historia de un intento de modernización de los Servicios de Inteligencia*
}

\author{
Carlos Ruíz Miguel
}

Arbor CLXXX, 709 (Enero 2005), 121-150 pp.

Este trabajo quiere hacer un balance del primer servicio de inteligencia moderno de nuestra historia. En primer lugar, se examinan sus antecedentes en los momentos anteriores a la transición política, apreciando la mutación de la inteligencia española, que pasa de ser un órgano de militares para vigilar a civiles a ser un órgano con cada vez más civiles para vigilar a militares. En segundo lugar, se refiere la normativa reguladora de este servicio. Después, se expone el proceso de normalización del CESID, que se traduce en una cada vez mayor vocación exterior paralela a la progresiva presencia de España en las relaciones internacionales. En ese proceso también se advierte un continuado esfuerzo por coordinar de forma centralizada la inteligencia para darle mayor eficacia. Esta modernización se complementa con la racionalización de la estructura burocrático-administrativa del servicio de inteligencia y la atribución de un estatuto específico al personal a su servicio. El sexto aspecto aquí analizado es el estudio de las competencias que nuestro ordenamiento asigna al servicio de inteligencia, donde se aprecia la paradoja de ver el, en mi opinión, excesivo maniatado de los servicios de inteligencia haciendo más difícil (y meritoria) su tarea. La eficacia en la realización de sus competencias depende de la existencia de adecuadas garantías protectoras del servicio de inteligencia, un asunto al que se dedica la séptima parte del trabajo. Finalmente, se hace una referencia a las carencias y disfunciones que sufrió el siempre necesario control de estos servicios.

* He tratado de esta cuestión en mi libro Servicios de inteligencia y seguridad del Estado constitucional, Madrid, Tecnos, 2002, p. 174 ss. Después, también ha estudiado el CESID la obra de Antonio M. DÍAZ FERNÁNDEZ, Los servicios de inteligencia españoles, Madrid, Alianza, 2005, p. 188 ss., que, por cierto, no cita el primer libro mencionado. 


\section{Antecedentes}

El antecedente del CESID lo encontramos a finales de los sesenta con la aparición de un organismo que resultará muy importante en la historịa de los servicios de información españoles.

En 1968 la oposición a la dictadura encontró en las Universidades cada vez mayor apoyo y, ante esta situación, el Subsecretario de Educación, Alberto Monreal Luque, solicitó al Alto Estado Mayor apoyo para hacer frente a la subversión en la Universidad. Como el Alto Estado Mayor quería quedar desvinculado de esta nueva misión que implicaba una clarísima intervención en la política interior, el resultado fue una hijuela suya, un organismo que inicialmente dependería del Ministerio de Educación: era la Organización Contrasubversiva Nacional (OCN) y que se puso al mando del entonces comandante José Ignacio San Martín. En 1969, el nuevo organismo pasó a llamarse Servicio Central de Documentación (SECED). En 1972, y por presiones del jefe del SECED, se produjo una reorganización que en líneas generales se ha mantenido hasta el 2001: por un lado, se remodelaba la Presidencia del Gobierno, haciendo depender al SECED del Ministro subsecretario de la Presidencia ${ }^{1}$, por otro, el jefe del servicio quedaba nombrado con el cargo de Director general ${ }^{2}$.

El SECED nacía como un órgano formado y creado por «militares», pero con «apariencia civil» (dependía del Ministerio del Interior y, luego, de Presidencia) para vigilar a ciudadanos civiles en el interior. Este origen suponía un inevitablemente solapamiento con otros órganos de información: bien los servicios «civiles» de la Guardia Civil, bien los «militares» del Ejército. Por eso, ya desde este primer momento se planteó el problema de la coordinación entre los servicios. Desde 1973 este problema se planteó con gran crudeza, sobre todo entre el SECED y su matriz, los órganos de información del Ejército. Una solución provisional, que fracasó, fue la creación el 26 de septiembre de 1973 de una «Comisión

\footnotetext{
1 Decreto 511/1972, de 3 de marzo. El artículo 1 de este Decreto reformaba el art. 2.2 del Decreto 245/1968, de 15 de febrero (sobre estructura de la Presidencia del Gobierno). El precepto dispuso, por un lado, que «dependerá directamente del Ministro subsecretario (de la Presidencia) el Servicio Central de Documentación (...)». Además, por otro lado, establecía que «el Jefe del Servicio Central de Documentación será designado por Decreto aprobado en Consejo de Ministros, a propuesta del Ministro Subsecretario de la Presidencia del Gobierno".

2 Decreto 512/1972, de 3 de marzo.
} 
El CESID: Historia de un intento de modernización...

Nacional de Coordinación de la Información (para la Seguridad del Estado)», presidida por el Presidente del Gobierno e integrada por el vicepresidente del Gobierno, los ministros de la Gobernación, Información y subsecretario de la Presidencia y el Teniente general jefe del Alto Estado Mayor, con la presencia de los ministros militares cuando así se requiriera $^{3}$.

La muerte de Franco y el paso de la dictadura a la democracia tuvo su repercusión en la organización de los servicios de inteligencia. Esta nueva organización coadyuvó a cumplir otros fines, como el de asignar un nuevo papel a las Fuerzas Armadas en el que quedaran privadas de sus servicios de inteligencia interior y el de simplificar la multiplicidad de servicios en aras de una mayor eficacia. Fue así como nació el actual servicio de inteligencia español, el Centro Superior de Información de la Defensa (CESID).

\section{Creación y normativa reguladora: El miedo a legislar}

El principal servicio de información que existía en España, el CESID, se creó en 1977, después de la elección de las Cortes que elaboraron la Constitución, pero antes de aprobarse la Constitución. Antes de las elecciones a las Cortes, el vicepresidente del Gobierno y ministro de Defensa, Gutiérrez Mellado, convocó a Andrés Cassinello (director del SECED) y a Manuel Vallespín (jefe de la división de inteligencia del Alto Estado Mayor) y les comunicó la decisión del Gobierno de fundir sus dos organizaciones en una sola, para lo que les pidió que formaran una comisión paritaria encargada de elaborar un anteproyecto en el que se diera cuerpo al nuevo organismo. La Comisión, de seis miembros, empezó a trabajar a finales de la primavera de 1977 en lo que iba a ser el «Servicio de Inteligencia de la Defensa", pero ante la falta de acuerdo en algunos puntos, fue Gutiérrez Mellado el que dio el impulso decisivo al nuevo organismo, cuya denominación fue puesta por el presidente Suárez ${ }^{4}$. En efecto, al reestructurarse la Administración Central del Estado y crearse el «Ministerio de Defensa" en el que quedaron integrados los anteriores Ministerios del Ejército, Marina y Aire se estableció que «bajo la dependencia directa del titular del Departamento se crea el Centro Superior de Infor-

\footnotetext{
3 José Ignacio SAn Martín, Servicio especial, Barcelona, Planeta, 1983, p 49-50.

4 Francisco Medina, Las sombras del poder. Los secretos del CESID, Madrid, Espasa Calpe, 1996, p 69-74.
} 
mación de la Defensa, al que se incorporarán las funciones y organismos que se determinen ${ }^{5}$. El desarrollo de este precepto se produjo meses después cuando se aprobó el reglamento orgánico del Ministerio de Defensa. En dicho Reglamento se estableció la primera regulación del CESID ${ }^{6}$, que ha sido objeto de modificaciones después de la entrada en vigor de la Constitución y de la LO 6/1980 por la que se regulan los criterios básicos de la defensa nacional y la organización militar.

A diferencia de lo que ocurre en otras Constituciones ${ }^{7}$ de la Unión Europea (como la alemana ${ }^{8}$ o la austríaca ${ }^{9}$ ), la nuestra no contiene pronunciamientos expresos que sirvan de cobertura para los servicios de inteligencia. Esta circunstancia no es casual. El hecho de que la transición hiciera coincidir el proceso de elaboración de la Constitución (1977-1978) con el de la reestructuración de los servicios de inteligencia, así como la circunstancia de que entre los dirigentes del recién creado CESID hubiera personas que habían estado destacadas en Alemania (como Manuel Vallespín) ${ }^{10}$, hizo que el joven organismo realizara gestiones (naturalmente discretas) ante los constituyentes para que en el artículo más conveniente se abriera un portillo para tener garantías en su trabajo, a fin de evitar tener los problemas que, ya en esas fechas, se habían encontrado los servicios de inteligencia de otros países. El CESID fue escuchado, pero se le adujo lo excesivo de la pretensión de tener un reconocimiento en la misma Constitución y se alegó que eso debía ser tratado en una ley específica y en otras normas reglamentarias ${ }^{11}$. Se perdió así una magnífica oportunidad de dar una seguridad jurídica a la existencia y el funcionamiento de los servicios de inteligencia. En el fondo, en mi opi-

5 Art. 2.5 del Decreto 1558/1977, de 4 de julio.

6 Art. 21 del Decreto 2723/1977, de 2 de noviembre.

7 No puede, por tanto, afirmarse que «hablando en términos comparativos, puede decirse, sin temor a exagerar, que el mundo de los servicios de inteligencia resulta, todavía hoy, por completo opaco a las Constituciones escritas» (Miguel REVENGA SÁNCHEZ, "Servicios de inteligencia y derecho a la intimidad», Revista Española de Derecho Constitucional no 61 [2001], p. 59 ss., p. 62).

8 Artículos 73.10.b y 87.1.2 GG.

9 El artículo 52a de la Constitución austríaca dispone en su párrafo primero que «las comisiones competentes del Consejo Nacional elegirán sendas subcomisiones permanentes para la supervisión de cualesquiera medidas adoptadas en salvaguardia de las instituciones constitucionales, y de su capacidad operativa, así como las medidas que se adopten por los servicios de información para asegurar la defensa militar del país».

10 Joaquín BARdavío, Pilar Cernuda y Fernando Jáuregui, Servicios Secretos, Barcelona, Plaza y Janés, 2000, p. 209.

11 BARDAvío et al., Servicios Secretos, cit., p. 212. 


\section{El CESID: Historia de un intento de modernización...}

nión, lo que latía era un cierto complejo ante la realidad de los servicios secretos que, en el fondo, eran identificados vagamente con la «herencia del franquismo». Esto explica también que se evitara aprobar una legislación en el Parlamento.

Las normas más importantes que sirvieron para regular el CESID se dictaron en $1981^{12}, 1982^{13}, 1985^{14}, 1995^{15}$ y $1996^{16}$ Todas estas normas tienen carácter reglamentario, si bien una ley de 1989, al disponer que todo el personal del CESID tuviera un mismo régimen jurídico, llevó a cabo un reconocimiento legal indirecto del mismo ${ }^{17}$. Sólo después de los ataques terroristas a Estados Unidos del 11 de septiembre de 2001, se decidió dar una cobertura jurídica legal a los servicios de inteligencia con la creación del CNI.

\section{Naturaleza del CESID: El camino hacia la normalización y la centralización}

Un servicio de inteligencia es un órgano eminentemente político. Por eso, los cambios políticos se traducen en cambios en estos servicios. No puede ser igual un servicio creado para un Estado mayormente aislado de la escena internacional que un servicio para un Estado internacionalmente relacionado. Esto explica que la naturaleza originaria (1977) del CESID experimentara una modificación importante a partir de $1982^{18}$ (en la última etapa del Gobierno de Calvo Sotelo y siendo ministro de De-

12 Real Decreto 726/1981, de 27 de marzo.

13 Orden del Ministerio de Defensa 135/1982, de 30 de septiembre, por la que se re gula la estructura y relaciones que ha de mantener el Centro Superior de Información de la Defensa (OM-1982).

14 Real Decreto 2632/1985, de 27 de diciembre, por el que se regula la estructura interna y las relaciones del Centro Superior de Información de la Defensa. (RD-1985).

15 Reales Decretos 1169/1995, de 7 de julio y 1324/1995, de 28 de julio, por el que se establece el Estatuto del Personal del Centro Superior de Información de la Defensa.

16 Real Decreto 266/1996, de 16 de febrero, por el que se modifica la estructura orgánica básica del Centro Superior de Información de la Defensa.

17 Disposición final $8^{\mathrm{a}}$ de la Ley 17/1989, de 19 de julio, Reguladora del régimen del personal militar y profesional. No es cierto, por tanto, la afirmación de la Exposición de motivos del PLRCNI según la cual «la actual regulación» del CESID «está contenida en una pluralidad de disposiciones, ninguna de ellas de rango legal».

18 Orden 135/1982, de 30 de septiembre, por la que se regula la Estructura y Re laciones que ha de mantener el Centro Superior de Información de la Defensa (OER CESID). 
fensa Alberto Oliart). Esta nueva orientación, realizada a partir del «informe Monzón» que pretendía crear sobre los cimientos del viejo CESID un nuevo servicio secreto, al estilo occidental, con dependencia del Ministerio de Exteriores y con un civil como director ${ }^{19}$, no creo que casualmente, coincide con el ingreso de España en la OTAN. Si inicialmente, el CESID surge orgánicamente ("de») y funcionalmente ("para») vinculado al Ministro de Defensa ${ }^{20}$, a partir de 1982, experimenta un cambio importante y, en gran medida, distorsionador.

Estas dos etapas se pueden apreciar en el tipo de misiones que cumplió el CESID en estas dos etapas: 1977-1982 y 1982 hasta su sustitución por el CNI. En sus primeros años, desde 1977, el CESID va a tener como prioridad la información militar, principalmente orientada hacia el interior de los propios Ejércitos, con el objetivo de conjurar eventuales conspiraciones de involución del régimen constitucional. Sin embargo, después del golpe de 23 de febrero de 1981, el CESID ampliará sus objetivos. Esta segunda fase, encuentra su consagración en la Orden de 1982, en la que, además de precisar su función de inteligencia militar ${ }^{21}$, se incluyen otras misiones. Así, en primer lugar, se amplían sus competencias de inteligencia interior, que no sólo abarcan ya a eventuales procesos involucionistas militares, sino también los de carácter terrorista («obtener, evaluar y difundir la información relativa a los procesos internos que, mediante procedimientos anticonstitucionales, atenten contra la unidad de la patria y la estabilidad de sus Instituciones fundamentales»); en segundo lugar, se le asignan por primera vez misiones de inteligencia exterior («obtener, evaluar y difundir la información necesaria para prevenir cualquier peligro, amenaza o agresión exterior contra la independencia o integridad territorial de España y para asegurar sus intereses nacionales», abarcando «los campos político, económico, tecnológico y militar»); y, en tercer lugar, se le atribuyen tareas de contrainteligencia («oponerse al espionaje y a las actividades de los servicios de inteligencia extranjeros que atenten contra la seguridad o los intereses nacionales, mediante su prevención, detección y neutralización dentro y

\footnotetext{
19 Fernando RUEDA, La Casa. El CESID: agentes, operaciones secretas y actividades de los espías españoles, Madrid, Temas de Hoy, 1993, p. 28.

20 Art. 21.1. y 2 del Real Decreto 2723/1977.

21 La OERCESID habla de «velar por la seguridad de la información, tecnología, procedimientos, objetivos e instalaciones de interés para la defensa, tanto propios como de los países aliados de España, en lo que no sea de competencia directa de las Fuerzas Armadas».
} 


\section{El CESID: Historia de un intento de modernización...}

fuera del territorio nacional») ${ }^{22}$. Esta extraordinaria ampliación de sus misiones provocará numerosas colisiones con otros servicios cuya solución no será sencilla.

Aunque se mantiene su dependencia orgánica del Ministro de. Defen$\mathrm{sa}^{23}$, funcionalmente se introduce una importante novedad pues a partir de ese momento el CESID trabajará «para» el Ministro de Defensa y también para el Presidente del Gobierno ${ }^{24}$. Esta doble dependencia funcional introduce un elemento de extraordinaria perturbación pues implica que al CESID se le asignan funciones de carácter «central» (las de inteligencia para el Presidente del Gobierno) que, sin embargo, no pueden ser realizadas idóneamente por cuanto orgánicamente el CESID es un servicio «periférico» sin competencias para supervisar lo que hagan otros servicios de inteligencia con los que se mantiene en posición de paridad orgánica.

El antiguo CESID, por tanto, fue configurándose como un órgano mixto con una naturaleza compleja entre la de un órgano central y un órgano periférico de inteligencia. Esto explica que tuviera encomendadas tres tipos de tareas de coordinación de la inteligencia en España. Las dos primeras se articulaban en torno a la materia de defensa. Así, según la primera, el CESID era «el órgano de información del Presidente del Gobierno, para el ejercicio de sus funciones de dirección de la política de defensa y de coordinación de la acción del Gobierno en la defensa del Estado»; y, en virtud de la segunda, era el órgano de información «del Ministro de Defensa, en el ejercicio de las funciones que le corresponden en materia de defensa y de política militar ${ }^{25}$. La tercera de ellas, sin embargo, revestía un carácter distinto porque tenía un carácter transversal y podía afectar a muy diferentes servicios. En efecto, al CESID se le asignaba la tarea de «coordinar la acción de los distintos organismos que utilicen me-

22 Art. 3 OERCESID.

23 Art. 2 OERCESID («EI Centro Superior de Información de la Defensa depende orgánicamente del Ministro de Defensa»).

${ }^{24}$ Art. 1 OERCESID («El Centro Superior de Información de la Defensa es el órgano encargado de satisfacer las necesidades de información del Presidente del Gobierno para el ejercicio de las funciones de dirigir y coordinar la acción del Gobierno en materia de de fensa, y del Ministro de Defensa para la ordenación y coordinación inmediatas de dicha política y la ejecución de la política militar»). En consecuencia, no está en lo cierto Medina cuando afirma que este cambio se produce "gracias a un Real Decreto" (fue con una Orden Ministerial) en «enero de 1984» (fue en septiembre de 1982),cfr. MEDINA, Los secretos..., cit., p. 74.

25 Art. 5.1 RDEOBMD. 
dios o procedimientos de cifra, garantizar la seguridad criptográfica, promover la adquisición coordinada de material y formar al personal especialista ${ }^{26}$. En consecuencia, toda la inteligencia de defensa o criptográfica era coordinada por el CESID.

\section{Estructura: Proceso de racionalización}

La estructura del CESID tenía en su cúspide al Director, subordinado al cual se encontraba la Secretaría General, a los que se añadían una serie de unidades de tipo diverso.

IV.1. El Director, ya en la época del CESID, era nombrado por el Gobierno, a propuesta del Ministro de Defensa ${ }^{27}$, habiendo pasado de tener rango de «Director general» a «Secretario de Estado» en la última etapa del CESID. La argumentación ofrecida para tal cambio fue la «conveniencia de facilitar las relaciones del Director (del CESID) con los órganos superiores de la Administración del Estado»28. El cambio tenía importancia por diversos motivos. En primer lugar, porque ello le habilitaba para ostentar por delegación expresa de su Ministro (el de Defensa) la representación de éste «en materias propias de su competencias, incluidas aquéllas con proyección internacional», sin perjuicio de lo que dispusieran las normas de Derecho Internacional ${ }^{29}$. En segundo lugar, porque podía asistir a las reuniones del Consejo de Ministros, cuando fuese convocado ${ }^{30}$. En tercer lugar, porque aunque el Director del CESID se hallase en un Departamento ministerial distinto, sí parecía prefigurarse una intención de situar al CESID o, al menos, a su Director, como coordinador de todos los servicios, quizá siguiendo el modelo norteamericano que atribuye al Director of Central Intelligence la doble tarea de coordinar los servicios de inteligencia y, a la vez, ser el jefe de la CIA. Esta posibilidad, se reforzaba en la legislación posterior que hacía al Director del CNI miembro de la Comisión Delegada del Gobierno para la Inteli-

\footnotetext{
26 Art. 5.3 RDEOBMD.

27 Art. 7.1 OERCESID. Es un error, por tanto, afirmar como hace algún autor que «quedaba claro que el Director General del CESID sería nombrado por el Presidente del Gobierno, a propuesta del Ministro de Defensa» (RUEDA, La Casa, cit., p. 37).

28 Real Decreto 741/2001, de 29 de junio, por el que se otorga rango de Secretario de Estado al Director del Centro Superior de Información de la Defensa.

${ }^{29}$ Art. 7.2 de la Ley 50/1997, de 27 de noviembre, de organización, competencias y funcionamiento del Gobierno.

30 Art. 5.2 de la Ley 50/1997.
} 
El CESID: Historia de un intento de modernización...

gencia del Estado que tiene asignada la misión de coordinar los diversos servicios de inteligencia del Estado. Ahora bien, al formar parte también de la Comisión los responsables de los otros servicios, la supuesta posición central del Director del CNI queda anulada, pues él, en cuanto director del CNI, no tiene ninguna competencia sobre los demás servicios.

Aunque inicialmente, los mandatos del director no tenían limitación, en febrero de $1996^{31}$ se estableció que el mandato del Director no podía exceder de un período máximo de cinco años ${ }^{32}$, lo que no se cumplió escrupulosamente $^{33}$. La limitación es una medida que presenta la ventaja de impedir que alguien utilice el puesto para erigirse en un «contrapoder», pero tiene el inconveniente de que obliga a prescindir después de cinco años de eventuales directores que hayan resultado especialmente eficaces en su labor.

La relevancia de la figura del director queda en gran medida determinada por el modo en que consiga establecer relaciones personales con sus superiores. Así, en 1996 se dispuso que fueran miembros del «Consejo de Dirección del Ministerio» de Defensa (órgano asesor del Ministro en la dirección y administración del Departamento), entre otros, «los titulares de los centros directivos con dependencia directa del Ministro» ${ }^{34}$, lo que significaba que el Director general del CESID formaba parte del mismo. Esto se explicaba por la buena relación entre el entonces Director (Calderón) y el entonces Ministro (Eduardo Serra). En 2001, sin embargo, se prescribió que ningún Director general fuera miembro nato de este Consejo (lo que excluía al Director del CESID), aunque se le podrá convocar «en aquellas ocasiones en que el Ministro lo considere oportuno» ${ }^{35}$. El cambio se explica por la mala relación entre Calderón y el Ministro del momento (Trillo).

31 El contexto de la limitación fue el escándalo de las «escuchas del CESID» que salpicó al que llevaba entonces ya catorce años como Director, General Manglano. Parece que la idea de limitar el mandato se debe a Gustavo Suárez Pertierra.

32 Art.1.1 del Real Decreto 2632/1985, de 27 de diciembre, por el que se regula la estructura interna y las relaciones del Centro Superior de Información de la Defensa (RDEIRCSD), art. 9.1 PLRCNI.

${ }^{33}$ El director del CESID en aquel momento (17 de febrero de 1996), Javier Calderón, permaneció en su puesto más de cinco años, pues fue relevado por el actual director, Jorge Dezcallar el 30 de junio de 2001.

34 Art. 23 RDEOBMD.

35 Real Decreto 64/2001, de 26 de enero, por el que se modifica el Real Decreto 1883/1996, de estructura orgánica básica del Ministerio de Defensa, modificado por el Real Decreto 76/200, de 26 de enero. 
IV.2. La Secretaría General fue un órgano creado en 1995 que tenía como misión «la asistencia al Director general en el ejercicio de sus funciones, en particular en la coordinación de los órganos del Centro». Además, debía sustituir «al Director general en los casos de vacante, ausencia o enfermedad $\imath^{36}$. En 1996 se otorgó a la Secretaría general el nivel orgánico de Subdirección General.

IV.3. Además del Director y del Secretario General, el CESID se componía de tres tipos de unidades: de inteligencia, de apoyo y de seguridad.

Las unidades de inteligencia eran cuatro: inteligencia exterior, contrainteligencia, inteligencia interior y Economía y Tecnología ${ }^{37}$. A la de «inteligencia exterior» le correspondía «obtener, evaluar y difundir la información para prevenir cualquier peligro, amenaza o agresión exterior contra la independencia o integridad territorial de España asegurando sus intereses nacionales», todo ello abarcando los campos «político, económico y militar» ${ }^{38}$. A la de "contrainteligencia» se le encargaba "oponerse al espionaje y a las actividades de los servicios de inteligencia extranjeros que atenten contra la seguridad o los intereses nacionales, mediante su prevención, detección y neutralización dentro y fuera del territorio nacional ${ }^{39}$. A la de «Inteligencia interior» se le asignaba «obtener, evaluar y difundir la información relativa a los procesos internos que, mediante procesos anticonstitucionales, atenten contra la unidad de España y la estabilidad de sus instituciones» ${ }^{40}$. A la de "Economía y Tecnología» le ocupaba el «obtener, evaluar y difundir la información necesaria para prevenir cualquier peligro, amenaza o agresión exterior contra la industria y el comercio español de armamento y material de guerra y para asegurar los intereses nacionales en los campos de la economía y la tecnología de interés específico para la Defensa», así como "velar por la seguridad de la información, tecnología, procedimientos, objetivos e instalaciones de interés para la Defensa, tanto propios como de los países aliados de España ${ }^{41}$.

Las unidades de inteligencia contaban con el apoyo de varias unidades específicas: «apoyo operativo», «apoyo técnico» y «personal y servi-

\footnotetext{
36 Art. 2 del Real Decreto 1169/1996, de 7 de julio, por el que se modifica la estructura orgánica del Centro Superior de Información de la Defensa; art. 10.1 PLRCNI.

37 Art. 2.1 RDEOBCSD.

38 Art. 4 RDEIRCSD.

39 Art. 5 RDEIRCSD.

40 Art. 6 RDEIRCSD.

41 Art. 7 RDEIRCSD.
} 
El CESID: Historia de un intento de modernización...

cios». La función de las unidades de apoyo era la de "atender las «necesidades de medios humanos, materiales y económicos, así como de medios, procedimientos o técnicas especiales» ${ }^{42}$. Alguna de estas unidades (como la llamada "Agrupación de Apoyo Operativo»), que realizaba varios cometidos de gran importancia, se hizo famosa por el escándalo de la sustracción de algunos importantes documentos por el coronel José Alberto Perote.

Finalmente, la unidad de seguridad debía «velar por la protección de las personas del Centro y sus instituciones» así como asumir «la salvaguardia de su información y documentación así como de la seguridad del funcionamiento del Centro» ${ }^{43}$.

\section{El Estatuto de los miembros de los miembros del CESID: Hacia la especialización}

Aun cuando el CESID se hallaba encuadrado orgánicamente en el Ministerio de Defensa, su personal tenía procedencia civil y militar. Los miembros del CESID se definían como quienes, «en virtud de nombramiento legal y una vez superadas las correspondientes pruebas de selección, se incorporan al mismo con una relación estatutaria de servicios profesionales y retribuidos con cargo a los presupuestos generales del Estado». El personal del Centro, tanto fuesen civiles como militares o guardias civiles, desde el momento en el que entraba al servicio del CESID, se regía por la lex specialis que es el Estatuto del CESID ${ }^{44}$, en lugar de la lex generalis de su estatuto de origen.

V.1. El ingreso en el Centro se efectuaba tras la realización de determinadas pruebas. Quien superase tales pruebas se incorporaría, con carácter temporal, al Centro. En este período, el personal se sometería a un proceso de formación y de prueba (de un período máximo de entre $1 \mathrm{o}$ 2 años, según la categoría del puesto), tras lo cual se efectuaría una valoración de la idoneidad del agente. La superación del período de valoración de idoneidad «supondrá la prestación de servicios en el Centro, en régimen de compromiso temporal, por un plazo mínimo de cuatro años, computándose a estos efectos el tiempo desde su ingreso». Desde el momento en que una persona entraba al servicio del CESID el Jefe directo

\footnotetext{
${ }^{42}$ Disposición adicional $7^{\mathrm{a}} \mathrm{RDEOBMD}$.

${ }^{43}$ Art. 2.2 RDEOBCSD.

44 Art. 1 EPCESID.
} 
del interesado realizaba anualmente un Informe «que permita apreciar las cualidades, méritos, aptitudes, competencia, forma de actuación profesional y eficacia en el desempeño del puesto de trabajo». Si el análisis de los informes anuales es positivo, a partir del tercer año de servicio al empleado en calidad temporal se le ofrece la posibilidad de integrarse en el Centro de forma permanente. Esta relación permanente se adquiere en virtud de nombramiento otorgado por el Ministro de Defensa ${ }^{45}$.

V.2. El cese del personal del CESID se producía por varias causas, algunas de carácter político, otras de tipo profesional y, finalmente, las de tipo discrecional. Las causas de orden político son la "pérdida de la nacionalidad española», el haber sido "elegido por las Cortes Generales para formar parte de los órganos constitucionales u otros cuya elección corresponda a las Cámaras», el ser «adscrito a los servicios del Tribunal Constitucional, del Defensor del Pueblo o del Tribunal de Cuentas» y el ser «nombrado miembro del Gobierno o de los órganos de gobierno de las Comunidades Autónomas, Corporaciones locales o alto cargo de las mismas». Las causas de tipo profesional iban desde el cese a petición propia a la jubilación, pasando por la separación del servicio por causas disciplinarias o penales o la no superación de las pruebas requeridas para acceder a la condición de personal temporal o permanente ${ }^{46}$. Finalmente, y con carácter general, se establecía que «el personal designado para ocupar un puesto del Centro podrá ser cesado (recte, destituido) libremente en el desempeño del mismo» debiéndose motivar la competencia para adoptar tal resolución de destitución ${ }^{47}$.

V.3. El Estatuto establecía también los derechos de los miembros, entre los que destacaban dos: el «obtener la protección y el respaldo del Centro respecto a su actuación regular como miembro del mismo, asumiendo aquél la responsabilidad patrimonial en que pudiera haber incurrido por las acciones $\mathrm{u}$ omisiones que le hayan sido legítimamente ordenadas, haciéndose cargo de la oportuna asistencia letrada»; y el «ser informado, al incorporarse a su puesto de trabajo de los fines, organización y funcionamiento de su unidad (...) y de las atribuciones, deberes y responsabilidades que le incumben ${ }^{48}$. Es importante advertir que el CESID únicamente sólo se obligaba a asumir la responsabilidad patrimonial y la defensa letrada por la actuación regular de un agente que le hubie-

\footnotetext{
45 Arts. 12-16 EPCESID.

46 Art. 18 EPCESID.

47 Art. 4.3 EPCESID.

48 Art. 25.c. y d. EPCESID.
} 
El CESID: Historia de un intento de modernización...

ra sido legitimamente ordenada. Esto significaba que de esta forma el CESID quedaba a salvo de responsabilidad en los casos en los que sus agentes realizasen actos contrarios al ordenamiento jurídico. En la práctica, sin embargo, la asistencia era mayor y el CESID no dejó de prestar su apoyo a agentes implicados en actuaciones que provocaron la incoación de procesos judiciales. Así, por ejemplo, en el primer «caso de las escuchas del CESID» ${ }^{49}$.

V.4. Los deberes del personal al servicio del CESID, presentaban algunos aspectos de especial interés por cuanto implicaban limitaciones de importantes derechos fundamentales.

Así, en primer lugar, el derecho a la intimidad de su personal queda afectado por su obligación de «evitar que su vida privada y pública ocasionen vulnerabilidades para las funciones encomendadas al Centro» ${ }^{50}$ y de facilitar información sobre sus bienes y derechos patrimoniales ${ }^{51}$.

En segundo lugar, las libertades de expresión e información quedaban limitadas por el deber que se asumía de "guardar el secreto profesional y estricta reserva sobre los asuntos que conozca sobre la organización, fuentes, medios, instalaciones y actividades del Centro, así como sobre la existencia y el contenido de esos documentos, identidades, objetos o elementos relacionados con los anteriores aspectos, de los que tenga conocimiento. Tampoco podrá revelarlos ni comunicarlos a ninguna persona, ni tenerlos en su poder sin la previa autorización expresa del Director». El reglamento disponía que «esta obligación tiene carácter permanente y por lo tanto será de aplicación con carácter indefinido incluso cuando se haya cesado en el Centro» ${ }^{52}$.

En tercer lugar, el personal se obligaba a guardar «la más estricta neutralidad política y sindical, acomodando sus actuaciones y conducta, en lo referente a la prestación del servicio, al superior interés nacional, obrando por encima de criterios e intereses propugnados por grupos sociales, políticos, económicos o religiosos». En consonancia con este deber,

49 SAP Madrid, Sección $15^{\text {a }}$, de 26 de mayo de 1999 (ponente: Andrés).

50 Art. 37.2.f. EPCESID.

51 Art. 41 EPCESID («El Centro podrá exigir a su personal cuanta documentación (sobre bienes y derechos patrimoniales) considere oportuna y en concreto la relativa a los siguientes extremos: a) Las declaraciones tributarias correspondientes al Impuesto sobre la Renta de las Personas Físicas y al Impuesto Extraordinario sobre el Patrimonio. b) Los bienes y derechos patrimoniales que posean. c) Los valores o activos financieros negociables. d) Las participaciones societarias. e) El objeto social de las sociedades de cualquier clase en las que tenga intereses").

52 Art. 38 EPCESID. 
«al personal del Centro le será de aplicación la prohibición que las normas reguladoras del régimen del personal militar establecen con respecto a la sindicación, ejercicio del derecho de huelga y asociación con finalidad reivindicativa o política» ${ }^{53}$. Esta limitación de los derechos fundamentales resultaba especialmente problemática más que por razones de fondo (pues no sería difícil argumentar la necesidad y proporcionalidad de la misma), por razones formales, dado que no estaba prevista en una ley ${ }^{54}$, pues figuraban en un reglamento administrativo que traía causa de una ley (la 17/1989) con un apoderamiento excesivamente amplio. Esta insuficiencia legal se trató de suplir con la prestación del consentimiento por los afectados ${ }^{55}$. No obstante, seguían existiendo obstáculos insalvables, a falta de ley, en aspectos como la limitación del derecho de sindicación y huelga que, según la ley que regula los mismos, no pue den ser renunciados para no obtener trato discriminatorio (art. 12 de la Ley Orgánica de Libertad Sindical).

V.5. El Estatuto fijaba también el régimen disciplinario de los miembros del Centro que se aplicaba con preferencia sobre otros regímenes a los que pudieran estar sometidos algunos miembros del mismo (militares, guardias civiles). Es importante advertir que, pese a que el CESID se hallaba encuadrado en el Ministerio de Defensa y una parte importante de sus miembros eran militares, su régimen disciplinario fijaba como Derecho supletorio «lo dispuesto en la legislación vigente de personal civil funcionario» ${ }^{56}$. Respecto al régimen disciplinario cabía introducir objeciones similares a las que se plantearon respecto a las restricciones en los derechos fundamentales que suponían ciertos deberes impuestos a los miembros del CESID. En efecto, el art. 25.1 CE establece el principio de legalidad en materia sancionatoria y, sin embargo, este régimen sancionador se hallaba contenido en una norma reglamentaria, de escasa cobertura legal (la ley 17/1989). Ciertamente, la jurisprudencia constitucional ha reconocido que «el alcance de dicha reserva de Ley pierde parte de su fundamentación en el seno de las relaciones de sujeción es-

53 Art. 37.1 EPCESID.

${ }^{54}$ De entre la abundante jurisprudencia constitucional pueden destacarse por su afán de sistematicidad de la teoría de la limitación de los derechos fundamentales las SSTC 66/1995, de 8 de mayo (ponente: Viver), FJ $4^{\circ}$ y $5^{\text {o }}$; y 207/1996, de 16 de diciembre (ponente: Gimeno) FJ $4^{\circ}$.

55 En Medina, Las sombras..., cit., p. 290-291, aparece un documento con una serie de renuncias que, según el autor, era firmado por todos los que entran a prestar servicios en el CESID.

56 Art. 42.3 EPCESID. 
El CESID: Historia de un intento de modernización...

pecial, aunque incluso en dicho ámbito una sanción carente de toda base legal devendría lesiva del derecho fundamental que reconoce el art. 25.1 de la Constitución $»^{57}$. La pregunta es ¿el régimen sancionador se hallaba «carente de toda base legal»? La jurisprudencia parecía haber aceptado que la alusión al CESID realizada en la ley de 1989 que estableció que el régimen disciplinario "se determinará en función del carácter y naturaleza del Centro» ${ }^{58}$ proporcionaba la base legal requerida ${ }^{59}$.

\section{Competencias: El Guardaespaldas maniatado}

Para cumplir sus misiones el CESID, como los demás órganos de inteligencia, tenía asignadas tres competencias básicas: obtener, analizar y proporcionar información. Sin embargo, el ordenamiento español, presa de unos complejos explicables por las circunstancias de la transición a los que antes se aludió, obstaculizó más allá de lo razonable al CESID en el ejercicio de esas competencias.

VI.1. Como es sabido, la obtención de información se lleva a cabo por múltiples vías y fuentes. La mayor parte de la información se obtiene por fuentes públicas, y el resto en fuentes no públicas. Para acceder a esta información no pública a su vez hay varios medios. Por un lado, existen medios «convencionales» o «formalizados»: las escuchas telefónicas y los registros domiciliarios son los principales. Pues bien, los tribunales españoles dieron una interpretación excesivamente rigorista a nuestro ordenamiento de suerte que el CESID quedó privado de poder obtener información por estas vías.

Hasta la aprobación de la nueva legislación del CNI, la posición del TS y del TC parecía sostenerse sobre la tesis de que el único fin legítimo para intervenir en las comunicaciones era la "seguridad pública». De aquí se derivaba que sólo pudieran instar esas medidas limitadoras los Cuerpos y Fuerzas de Seguridad pues a ellos se les encomendaba «el mantenimiento

57 STC 219/1989, de 21 de diciembre (ponente: García-Mon), FJ $2^{\circ}$.

58 Disposición final $8^{\mathrm{a}}$.d de la ley 17/1989.

59 SAN, sección $5^{\text {a }}$, de 22 de julio de 1999 (ponente: Gil Sáez), recurso 1874/1996, FJ $6^{\circ}$ («cabe advertir que el Estatuto está aprobado por el Real Decreto 1324/1995, de 28 de julio, trae causa directa de la Disposición final octava de la Ley 17/1989, de 19 de julio, sin que conste que contra aquella norma reglamentaria, bien en su totalidad, bien respecto de alguna de sus disposiciones [...] se interpusiera recurso contencioso-administrativo directo»). 
de la seguridad pública» (art. 1.4 LOFCS). Desde esta perspectiva, sólo la JIGC (Jefatura de Información de la Guardia Civil), la CGICNP (Comisaría General de Información del Cuerpo Nacional de Policía) y los eventuales órganos de inteligencia autonómicos (encuadrados en sus respectivas policías) y en el marco de investigaciones criminales podrían solicitar la autorización para tales intervenciones, mientras el CESID y los servicios de información militares (que no son fuerzas de "seguridad») quedaban privados de esta posibilidad. Esta idea de que la «seguridad pública» es el fin legítimo perseguido por estas medidas limitadoras (registros domiciliarios y escuchas telefónicas) subyace, aun cuando no aparezca expresamente, en la jurisprudencia del $\operatorname{TS}^{60}$ y es defendida por autores como Gimbernat ${ }^{61}$. También apoyaba esta idea el TC. Inicialmente obiter dictum ${ }^{62}$, se refirió a la intervención en las comunicaciones como «diligencia de investigación». Más tarde profundizó en esta idea al decir que «la garantía jurisdiccional del secreto de las comunicaciones no se colma con su concurrencia formal -autorización de procedimiento de un órgano jurisdiccional- sino que ésta de estar dictada en un proceso, único cauce que permite hacer controlable, y con ello jurídicamente eficaz, la propia actuación judicial». De esta suerte, la garantía sobre las medidas de intervención de las comunicaciones tomadas en un momento anterior al proceso existe cuando las mismas "se unen, pese a todo, sin solución de continuidad, al proceso judicial incoado en averiguación del delito, satisfaciendo así las exigencias de control del cese de la medida que, en otro supuesto, se mantendría en un permanente y, por ello constitucionalmente inaceptable, secreto» ${ }^{63}$. El hecho de que el CESID no sea una "policía» cuyas investigaciones abren un "proceso» provocó, en primer lugar, que el director del CESID, Manglano, hubiera solicitado la inclusión de su servicio en la LOFCS (para beneficiarse de esos instrumentos legales) y, en segundo lugar, que de hecho el CESID no solicitase intervenciones de ese carácter ni tribunales como la Audiencia Nacional se las faciliten ${ }^{64}$.

60 SSTS (2a) de 12 de septiembre de 1994 (ponente: García Miguel), FJ 20; de 20 de diciembre de 1996 (ponente: Soto), FJ $3^{\circ}$. Cfr. también STS $\left(2^{\mathrm{a}}\right)$, de 22 de marzo de 2001 (ponente: Martín Pallín), FJ $8^{\circ} .3$.

61 Defiende esta postura, Enrique GIMBERNAT ORDEIG, «Escuchas telefónicas y reforma del CESID», El Mundo, 15 de junio de 1999, p. 16-17; IDEM, «Escuchas telefónicas y estado de necesidad", El Mundo, 4 de mayo de 1998, p. 4-5.

62 SSTC 86/1995, de 6 de junio (ponente: Gimeno) FJ 3º 49/1996, de 27 de abril (ponente: Jiménez de Parga), FJ $3^{\circ}$.

63 STC 49/1999, de 5 de abril (ponente: Vives), FJ $6^{\circ}$.

64 RuedA, La Casa, cit., p. 351-352, 355. 
El CESID: Historia de un intento de modernización...

Junto a estos mecanismos «formalizados» de obtención de información no pública, los servicios de inteligencia suelen utilizar fuentes no abiertas al público y acciones encubiertas. Este tipo de información se obtiene por métodos no convencionales a los que el ordenamiento jurídico apenas hace referencia.

Este tipo de técnicas estaban indirectamente aludidas en la normativa reguladora del CESID que encomendaba a las unidades de apoyo realizar las acciones que «requirieran medios, procedimientos o técnicas especiales»" ${ }^{65}$ (que debían estar "previa y debidamente autorizadas») y facilitar la infraestructura y el apoyo necesarios a aquellas actividades del Centro «que precisaran el empleo de personas, instalaciones y medios extraordinarios». Estas dos tareas se correspondían con lo que más tradicionalmente se suele calificar de "espionaje» y que por la amplitud de sus términos podían abarcar desde técnicas, en principio, legales (como la utilización de espías o agentes encubiertos) hasta medios que, eventualmente, podían ser de dudosa legalidad o incluso claramente ilegales. El problema es si una norma reglamentaria puede legitimar la utilización de este tipo de técnicas, máxime cuando, en el caso de los servicios de información policiales, estas posibilidades han sido introducidas me diante una ley orgánica.

Junto a las técnicas «clásicas» de obtención «no convencional» de información, las unidades de apoyo del antiguo CESID establecían y explotaban «los medios de comunicación del Centro» así como planificaban y dirigían «la adquisición de información por procedimientos y medios técnicos». Igualmente, les cumplía el «criptoanalizar y descriptar por procedimientos manuales, medios electrónicos y criptofonía», así como el «realizar investigaciones tecnológico-criptográficas y formar al personal especializado en criptología» ${ }^{66}$.

VI.2. Para la realización de todas estas actividades los servicios de inteligencia requerían una serie de fondos. Una parte de los mismos se hallaba consignada en la correspondiente partida que la Ley de Presupuestos Generales del Estado establecía para cada organismo ${ }^{67}$, pero existía otra parte que se obtenía de los fondos reservados ${ }^{68}$. Hasta 1995, la regulación sobre el uso de los fondos reservados tenía importantes la-

65 Art. 2.2 RDEOBCSD.

66 Art. 11.1 RDEIRCSD.

67 Para el año 2001 al CESID se le asignaron en los presupuestos generales del Es tado 17.640 millones de pesetas.

68 El CESID dispone para el año 2001 de 1.400 millones en fondos reservados. 
gunas que, unidas a la trascendencia que tuvo en la opinión pública el llamado caso fondos reservados, originó que en 1995, se procediera a la regulación de estos fondos por la correspondiente ley ${ }^{69}$. Como ha sostenido el TS, «tales fondos constituyen una partida presupuestaria que, aprobada por las Cortes Generales y excluida por su propia naturaleza y destino de cualquier control, queda vinculada a los fines que constitucional y legalmente son propios del Ministerio a quien se encomienda su gestión» ${ }^{70}$.

A partir de la ley de 1995, tenían la consideración de fondos reservados «los que se consignen como tales en las Leyes de Presupuestos Generales del Estado y que se destinen a sufragar los gastos que se estimen necesarios para la defensa y seguridad del Estado». Los gastos reservados «se caracterizan respecto a los demás gastos públicos por la prohibición de publicidad ("toda la información relativa a los créditos destinados a gastos reservados, así como la correspondiente a su utilización efectiva, tendrán la calificación de secreto, de acuerdo con las leyes vigentes en materia de secretos oficiales» ${ }^{71}$ ), por estar dotados de un especial sistema de justificación y control» ${ }^{72}$ y porque los únicos Ministerios en los que pueden consignarse estos fondos son Asuntos Exteriores, «Justicia e Interior» y Defensa ${ }^{73}$. Aunque en su tramitación no requieren justificación documental, se intenta evitar la arbitrariedad exigiendo el previo informe del Interventor general de la Administración del Estado. En su uso se deben dictar las normas internas necesarias para asegurar que el uso de los fondos procedentes de los créditos de gastos reservados se realiza, por las autoridades del Estado a quienes se les asignen, únicamente para financiar las actividades de defensa y seguridad del Estado ${ }^{74}$.

El destino de los fondos reservados, por tanto, era únicamente el de «financiar las actividades de defensa y seguridad del Estado». Lo más habitual es que el uso de estos fondos sirviese para financiar ciertas actividades de los servicios de inteligencia en el cumplimiento de los citados fines.

69 Ley 11/1995, de 11 de mayo, reguladora de la utilización y control de los créditos destinados a gastos reservados (LGR).

70 STS $\left(2^{\mathrm{a}}\right)$, de 12 de marzo de 1992 (ponente: Vega), FJ $13^{\circ}$.

71 Art. 3 LGR.

72 Art. 1 LGR.

73 Art. 4 LGR. La referencia a "Justicia e Interior» se explica porque en el momento de dictarse la ley se había producido la unificación de ambos departamentos, que se disolvió después. Aunque lo lógico sería que sólo Interior pudiera disponer de ellos, la dicción literal hace difícil excluir a Justicia.

74 Arts. 5 y 6 LGR. 
El CESID: Historia de un intento de modernización...

Sin embargo, es perfectamente posible, y está admitido en nuestro ordenamiento, que tales fondos financien actividades de defensa y seguridad del Estado realizadas por particulares. Este fue el argumento del caso Crillon. Este caso se originó tras la denuncia del ex-director general de la Guardia Civil, Luis Roldán, contra el ex-ministro de Defensa García Vargas y el ex-vicepresidente del Gobierno Narcís Serra, que utilizaron fondos reservados para pagar a una agencia internacional de detectives privados especializada en inteligencia económica (la agencia Kroll), a fin de que investigara las actividades del ex-presidente de Banesto, Mario Conde. La denuncia de Roldán fue archivada por el Tribunal Supremo ${ }^{75}$. Según el tribunal, la utilización de fondos reservados para efectuar esa investigación no suponía un «uso indebido» de los mismos, ya que el conocimiento de las actividades del presidente de un banco, ante la sospecha de que pudiera estar cometiendo irregularidades que podrían provocar «graves riesgos en el sistema financiero nacional», "pretende salvaguardar intereses públicos relevantes que se pueden conectar perfectamente con la seguridad pública general». La doctrina sentada por el TS, antes de la entrada en vigor de la LGR, siguió siendo válida después de la misma. La propia esencia de la actividad de información exige en muchas ocasiones realizar pagos a individuos particulares o sociedades de derecho privado que realizan ciertas actividades de inteligencia que el Estado no puede llevar a cabo con la misma efectividad. Así ha ocurrido en los EE.UU. desde hace mucho tiempo ${ }^{76}$. En consecuencia, perfectamente admisible era el pago de fondos reservados a individuos o sociedades particulares, siempre y cuando se cumpliesen los fines establecidos en la ley.

\section{Las garantías de los servicios de los servicios de inteligencia: La represión pública de la revelación de lo secreto}

Los servicios de inteligencia sólo pueden ser eficaces si se preserva el secreto de su organización y funcionamiento. Para que ello sea así, el poder público establece ciertas garantías de que el secreto no saldrá de esa esfera bien para llegar a la luz pública bien para llegar a otras esferas secretas no autorizadas.

75 Auto del Tribunal Supremo (2a), de 25 de julio de 1995 (ponente: Martín Pallín).

76 Recordemos que ya el caso Curtiss-Wright, enjuiciado por el TS, versaba sobre el pago de fondos reservados a un espía "privado», en la Guerra Civil norteamericana. 
VII.1. La primera de las garantías es de carácter constitucional-administrativo y consiste en la técnica de la clasificación. La regulación de la misma se basa en la ley de secretos oficiales de 1968 (modificada en 1978) y desarrollada por un reglamento de $1969^{77}$. El sometimiento al régimen de secreto de una materia puede realizarse por dos vías: legislativa o ejecutiva. Así, por una parte, "tendrán carácter secreto, sin necesidad de previa clasificación, las materias así declaradas por Leyn ${ }^{78}$. El ejemplo más claro de ello, en el que parece haber acuerdo de la doctrina, es el de la ley sobre fondos reservados ${ }^{79}$. Por otra parte, la limitación de la publicidad puede efectuarse a través de un acto singular de «clasificación".

La naturaleza del acto de clasificación, y la consiguiente discusión acerca de si es un "acto político» ${ }^{80}$ o un "acto administrativo» ha sido muy polémica ${ }^{81}$. Frente a estas dos opiniones unilaterales, cabría considerar que el acto clasificatorio es un acto mixto, lo cual parece encajar en la nueva ley de jurisdicción contenciosa. Según la misma, son objeto de control por los tribunales «los elementos reglados» en relación con «los actos del Gobierno o de los Consejos de Gobierno de las Comunidades Autónomas, cualquiera que fuese la naturaleza de dichos actos» ${ }^{82}$. De esta suerte la clasificación tendría una faceta administrativa, encarnada en una serie de elementos formales de tipo reglado contenidos en la propia LSO, a saber, la necesidad de que su emisión provenga sólo de determinados sujetos (Consejo de Ministros y Junta de Jefes de Estado Mayor), la necesidad de unas determinadas formalidades en su publicación, y la necesidad de motivación. Sin embargo, más allá de estos elementos, nos encontramos con la faceta política del acto clasificatorio. No obstante, aunque esa creemos que es la solución más razonable no es la que ha ad-

\footnotetext{
77 Ley 9/1968, de 5 de abril, reguladora de los secretos oficiales (LSO), reformada por la ley 48/1978, de 7 de octubre. Reglamento de la Ley de Secretos Oficiales, aprobado por Real Decreto 242/1969, de 20 de febrero (RSO).

78 Art. 1.2 LSO.

79 Íñigo Segrelles de AREnAZA, Protección penal del secreto de Estado, Madrid, Edersa, 1994, p. 38-39.

80 La categoría del «acto político» está admitida por la jurisprudencia constitucional en varias resoluciones (SSTC 45/1990, de 15 de marzo [ponente: Leguina], FJ 20; 220/1991, de 25 de noviembre [ponente: Díaz], FJ $2^{\circ}$ ).

81 Araceli MANJón-CABEZA Olmeda, «Secretos oficiales, ley y control judicial», $E l$ Mundo de 12 de octubre de 1996, p. 4-5.

${ }^{82}$ Art. 2.a de la Ley 29/1998, de 13 de julio, de la Jurisdicción Contencioso-Administrativa.
} 
mitido nuestro TS que solucionó de forma penosa el problema atribuyendo al acto de clasificación la categoría de «acto político» para, incongruentemente, darle el tratamiento de un «acto administrativo» revisable en todos sus extremos por los tribunales de lo contenciosoadministrativo.

La clasificación puede tener dos grados de protección: «secreto» («las materias que precisen del más alto grado de protección por su excepcional importancia y cuya revelación no autorizada por autoridad competente para ello, pudiera dar lugar a riesgos o perjuicios de la seguridad del Estado, o pudiera comprometer los intereses fundamentales de la Nación en materia referente a la defensa nacional, la paz exterior o el orden constitucional» ${ }^{83}$ y "reservado» ${ }^{84}$ que es una calificación con un perfil escasamente definido, pues ni los objetos de clasificación, ni el perjuicio que eventualmente pueda ocasionar su publicación son distintos de los cubiertos bajo la rúbrica de «secreto». La única diferencia es que la clasificación de "reservado» se aplica a materias de «menor importancia»" ${ }^{85}$. La consecuencia jurídica de la distinción entre ambas clasificaciones se produce a la hora del control parlamentario de las materias clasificadas, como bien vio García-Trevijano ${ }^{86}$.

El principal acto clasificatorio operado en nuestro ordenamiento se realizó mediante acuerdo del Consejo de Ministros de 28 de noviembre de 1986. En el mismo, se otorgó la clasificación de "secreto" a materias como: los procedimientos, técnicas y fuentes de los servicios de inteligencia; la Directiva de Defensa Nacional; las informaciones, análisis o evaluaciones de amenazas actuales o potenciales a la paz y la seguridad en España; el Plan General de la Defensa Nacional; las claves y material de cifra criptográfica; los informes y datos estadísticos sobre movimientos y fuerza, buques o aeronaves militares; etc. Por su parte, recibieron la clasificación de "reservado», entre otras, las siguientes materias: los destinos del personal de carácter especial; los planes de seguridad de instituciones y organismos públicos, así como de las unidades, centros u organismos de las Fuerzas Armadas y de los centros de producción de material de guerra; las plantillas de personal y de medios y de equipo de las unidades; etc.

83 Art. 3.I RSO.

84 Art. 3 LSO.

85 Art. 3.II RSO.

86 Ernesto García-TREviJANO GaRniCA, «Materias clasificadas y control parlamentario», Revista Española de Derecho Constitucional n 48 (1996), p. 145 ss., p. 164. 
Las consecuencias de la clasificación son varias. En primer lugar, restringe al acceso y la publicidad de las materias afectadas a los órganos y las personas debidamente facultadas para ello pudiéndose ordenar «la prohibición de acceso» y "limitaciones de circulación» a personas no autorizadas en locales, lugares o zonas en que radiquen las materias clasificadas ${ }^{87}$. En segundo lugar, obliga a toda persona a cuyo conocimiento o poder llegue cualquier "materia clasificada» a mantenerla en secreto y a entregarla a la autoridad civil o militar más cercana y, si ello no fuese posible, a poner en conocimiento de ésta su descubrimiento o hallazgo $0^{88}$. No obstante, al no estar sancionado el incumplimiento de esta obligación la misma ha sido ignorada en casos como el famoso de los «papeles del CESID» en el que materiales clasificados llegaron a manos de personas no autorizadas (banqueros o periodistas) sin que los mismos mantuvieran esa información en secreto ni la entregaran a la autoridad correspondiente. En tercer lugar, la clasificación de una materia obliga a la adopción de una serie de medidas de seguridad relativas a la custodia, traslado, transmisión, registro, inventario, examen y destrucción del material clasificado 89 .

VII.2. Junto a la «clasificación» existían otras garantías.

Una garantía, de orden penal, era la tipificación del delito de revelación de secretos. La garantía penal general del secreto de Estado se proyecta, con la máxima intensidad, sobre los funcionarios públicos, y de modo menos acentuado sobre los particulares.

En el ámbito jurisdiccional, el ordenamiento podía proteger la actividad de los servicios de inteligencia mediante la técnica del «secreto profesional» que permite excepcionar el deber de colaborar con la justicia que establece el art. $118 \mathrm{CE}$, cuyo incumplimiento está penado como delito de denegación de auxilio a la justicia (art. $412 \mathrm{CP}$ ). La cuestión se ha suscitado en la práctica en varias ocasiones. El art. 417.2 LECr. dice que «no podrán ser obligados a declarar como testigos», los funcionarios públicos «cuando no pudieren declarar sin violar el secreto que por razón de sus cargos estuvieren obligados a guardar, o cuando, procediendo en virtud de obediencia debida, no fueren autorizadas por su superior jerárquico para prestar la declaración que se les pida». Sin embargo, han existido casos en los que el TS decidió que el deber de colaboración con la justicia prevalecía sobre el secreto profesional, afirmando que la excep-

\footnotetext{
87 Art. 8 LSO.

88 Art. 9.1 LSO.

89 Arts. 13 a 32 RSO.
} 
ción del deber de colaboración con la justicia de los testigos puede ceder ${ }^{90}$. A nuestro juicio, en esas resoluciones el TS hizo unas argumentaciones sumamente cuestionables.

Los tribunales declinaron articular una garantía jurisdiccional consistente en rechazar como pruebas documentales aquellas consistentes en material clasificado como secreto en tanto en cuanto no sea desclasificado ${ }^{91}$. Pero esto creemos que fue una decisión equivocada porque si los jueces «no pudieran desconocer» las "pruebas» aportadas cualquiera que fuera su origen, incluso ilícito, se estaría abriendo la puerta al uso de cualesquiera procedimientos, por ilegales que fueran, para conseguir la victoria en el proceso.

\section{El control del CESID: cuando las buenas intenciones producen malos resultados}

El control de los servicios de inteligencia siempre ha sido una de las cuestiones más debatidas en el estudio de su régimen jurídico. También lo fue en el caso del CESID. Existen tres modos básicos de controlar un servicio de inteligencia: por el Ejecutivo, por el Parlamento o por los jueces.

VIII.1. El control más real y menos regulado ha sido el del poder ejecutivo. Contra la tesis de configurar a los servicios de inteligencia como una especie de órgano "técnico» de carácter «independiente» respecto al Gobierno, lo cierto es que el CESID se configuró, creemos que acertadamente, como un órgano dependiente del Ejecutivo de cuyos actos se hizo responsable al Ejecutivo. En la experiencia del CESID esto llegó a significar la dimisión del, en su momento, vicepresidente del Gobierno, Narcís Serra.

VIII.2. Junto al control por el propio poder ejecutivo, en el Derecho Comparado ha ido emergiendo una exigencia cada vez mayor de control por el Parlamento. La realidad es que en la historia del CESID este control ha sido, realmente, inexistente. El Congreso de los Diputados tenía una muy limitada capacidad de seguimiento regulada en una resolución de $1992^{92}$, que sustituía, de forma algo más generosa, a otra de $1986^{93}$ que había sido

$90 \operatorname{STS}\left(2^{\mathrm{a}}\right)$, de 22 de marzo de 1986; ATS $\left(2^{\mathrm{a}}\right)$, de 20 de febrero de 1995 , FJ $4^{\circ}$.b.

91 STS $\left(2^{a}\right)$, de 22 de marzo de 2001, FJ $6^{\circ} .7$.

92 Resolución de la Presidencia del Congreso de los Diputados de 2 de junio de 1992, sobre acceso a secretos oficiales.

93 Resolución de la Presidencia del Congreso de los Diputados, de 18 de diciembre de 1986, sobre acceso a materias clasificadas. 
juzgada como excesivamente restrictiva ${ }^{94}$. Esta resolución era una norma con rango de ley incardinada en el Reglamento del Congreso de los Diputados para cubrir una laguna del mismo. El mecanismo de control que disponía esta resolución se demostró, cuando menos, muy insuficiente ${ }^{95}$.

Junto a este mecanismo se discutió si podría servir como instrumento de control de los servicios de inteligencia la facultad que el Reglamento del Congreso de los Diputados garantiza a los diputados individualmente considerados de «recabar de las Administraciones Públicas los datos, informes o documentos que obren en poder de éstas». Según ese reglamento la Administración «deberá facilitar la documentación solicitada o manifestar al Presidente del Congreso, (...) las razones fundadas en derecho que lo impi dan ${ }^{96}$. Frente a la opinión de Cano Bueso que dejaba abierta la puerta a que incluso en los supuestos de información clasificada el Gobierno entre gara esa documentación a los diputados individualmente considerados ${ }^{97}$, creemos que era más acertada la posición de García-Trevijano, según la cual si la información solicitada por el diputado estaba clasificada, no es que la Administración requerida "pudiera" dejar de facilitarla, sino que "no podía» facilitarla ${ }^{98}$. En su opinión, tal acto denegatorio sólo podría ser objeto de un recurso de amparo ante el Tribunal Constituciona99. La exclusión de los diputados individuales del régimen especial de conocimiento de las materias clasificadas que operó la Resolución de la Presidencia del Congre so de los Diputados de 18 de diciembre de 1986 (antecedente de la de 2 de junio de 1992) fue recurrida en amparo ante el TC, pero éste rechazó el re curso por una cuestión formal sin entrar en el fondo del mismo ${ }^{100}$.

${ }^{94}$ Roberto L. Blanco VAldÉs, La ordenación constitucional de la Defensa, Tecnos, Madrid, 1990, p. 154.

95 Un profesor aporta pruebas contundentes de ello: Cfr. Juan CANO BuEso, «Información parlamentaria y secretos oficiales», Revista de las Cortes Generales 42 (1997), p. 30-34. Esta opinión se nos muestra manifiestamente mejor fundada que aquella que considera que "este esquema ha funcionado satisfactoriamente, o al menos sin conflictos»: Cfr. Pablo SANTOlaya MachetTi, «El control de los Secretos de Estado; la experiencia en Derecho comparado", Poder Judicial 40 (1995), p. 57 ss., p. 72 y 73. Sin conflictos, sin duda, pero satisfactoriamente es muy dudoso.

96 Art. 7 del Reglamento del Congreso de los Diputados.

97 CANO Bueso, "Información parlamentaria...», cit., p. 25.

98 GARcÍA-TREVIJANO, «Materias clasificadas...», cit., p. 175. Así se desprende también de la historia legislativa de esta resolución (CANO BUESO, «Información parlamentaria...», cit., p. 19).

99 García-TReviJano, «Materias clasificadas...», cit., p. 177.

100 STC 118/1988, de 20 de junio (ponente: Rodríguez-Piñero). 
El CESID: Historia de un intento de modernización...

Un problema importante y que pudo haber adquirido enorme gravedad es el de si el Senado tenía potestad para acceder a documentos clasificados. En principio, es indudable que el Reglamento del Senado no disponía nada sobre el particular y que tampoco esta laguna había sido suplida por el Presidente de la Cámara, de acuerdo con la Mesa. La cuestión se formulaba así: dado que el Reglamento no había determinado la forma de acceso a la documentación clasificada, ¿tenía acceso el Senado a la misma?. El problema se planteó inesperadamente cuando el Pleno del Senado acordó la creación de una Comisión de investigación sobre el GAL que muy poco después se disolvión ${ }^{101}$. Ante la negativa del Gobierno (del PSOE) a desclasificar los llamados «papeles del CESID», el Senado (con mayoría absoluta de PP) aprobó la creación de esta Comisión de investigación para que, una vez creada, la misma solicitara al Gobierno los citados «papeles». El Gobierno anunció su negativa a entregar los famosos «papeles» arguyendo que sólo el Congreso estaba facultado para verlos. La negativa del Gobierno podría haber generado un conflicto entre órganos constitucionales de no haberse disuelto la Comisión de investigación muy poco después.

Después de que se produjeran algunos escándalos relacionados con el uso de los fondos reservados, se dictó en 1995 una ley para controlar el uso dado a los "fondos reservados». Esa ley preveía controles parlamentarios sobre ese tipo de gastos reservados. Sin embargo, el Ejecutivo incumplió el deber de información semestral que la ley establecía para el Gobierno ante una Comisión secreta ${ }^{102}$.

En segundo lugar, con carácter anual y atendiendo en todo caso al carácter secreto de los fondos y a su régimen no público, la Comisión podrá elaborar un informe para su remisión a los Presidentes del Gobierno y del Tribunal de Cuentas sobre el uso dado a los fondos ${ }^{103}$. En tercer lugar, cuando cada Ministerio competente dictase la correspondiente normativa interna para asegurar que los fondos se destinaban a sus fines específicos, debería remitir la misma a la Comisión del Congreso ${ }^{104}$.

101 La Comisión fue creada por acuerdo del Pleno de la Cámara (dominada con mayoría absoluta del PP, frente al Congreso en el que había mayoría relativa del PSOE) el 18 de octubre de 1995. Veintitrés días después se decidió la disolución de esta Comisión.

102 En octubre de 1999, cuatro años después de entrar en vigor la Ley reguladora de los Gastos Reservados, la Comisión de Secretos Oficiales, encargada de vigilar los fondos reservados, sólo se había reunido en tres ocasiones.

103 Art. 7 LGR.

104 Art. 6 in fine LGR. También en este aspecto parece que hay que entender derogado el art. 6.3 de la Ley 13/1994, que dispone que «el acceso de las Cortes Generales a 
Cano Bueso puso de manifiesto los problemas que conllevaba la indefinición jurídica de esta "Comisión» de control de los fondos reservados, que no quedaba claro si seguía el régimen general de las Comisiones parlamentarias (lo que suponía que cabría tramitar ante la misma preguntas, comparecencias informativas, etc.) o uno especial ${ }^{105}$. Parecía más acorde con la naturaleza secreta y especial de los fondos reservados su consideración como un órgano especial.

VIII.3. Es, sin embargo, el ámbito de los tribunales el que ha sido más discutido como sede del control de los servicios de inteligencia. Esa intensa discusión también se produjo en relación con el CESID.

Los actos del CESID en materia de personal estuvieron sometidos al control jurisdiccional ${ }^{106}$. Aunque la ley que regula los fondos reservados no habla sobre la posibilidad de someter su uso a un control judicial, es lo cierto que los tribunales han efectuado tal tipo de control antes de la entrada en vigor de la LGR en 1995, y después. Antes de 1995, el TS tuvo ocasión de pronunciarse en dos ocasiones sobre los fondos reservados. Por un lado, en el caso Crillon, ya referido, el TS juzgó sobre la legalidad del destino dado a los fondos, analizando la cuestión de si era lícito el uso de fondos reservados para contratar a una empresa privada de inteligencia económica con la misión de espiar a un banquero español. Por otro lado, el TS tuvo ocasión de declarar que los fondos reservados pertenecen a la esfera del "secreto» que tienen obligación de respetar los funcionarios y autoridades ${ }^{107}$. La mayor parte de los casos, sin embargo, se refieren a hechos anteriores a la ley de 1995, pero sentenciados con posterioridad a esa fecha. Así ocurrió, en primer lugar, con el caso Marey, donde el TS dijo que «el que tales gastos o fondos reservados no hayan de ser justificados en cuanto a su aplicación concreta, precisamente por el secreto del fin o actividades a que se hallan destinados, no impide el que cuando se acredite un uso desviado de ese fin el hecho pueda ser constitutivo del delito de malversación de caudales públicos» ${ }^{108}$. En segundo lugar, los tribunales controlaron la legalidad de los gastos reservados en el caso

\footnotetext{
la información sometida al deber de secreto se realizará a través del Gobernador del Banco de España...».

105 CANO BUESO, «Información parlamentaria...», cit., p. 29.

106 SAN, sección $5^{\text {a }}$, de 22 de julio de 1997 (ponente: Gil Ibáñez); STSJ Madrid, sección $6^{\text {a }}$, de 23 de septiembre de 1999 (ponente Peña Elías), FJ 2. Existen más casos: SSAN, sección de 22 de julio de 1997 (ponente: Gil Sáez) en los recursos 1874 y 1875 de 1996. Cfr. También Arts. 50. 52 y 53 EPCESID.

107 STS $\left(2^{a}\right)$, de 12 de marzo de 1993 , FJ $13^{\circ}$.

108 STS $\left(2^{\mathrm{a}}\right)$ de 29 de julio de 1998 (ponente: Delgado García), FJ $18^{\circ}$.
} 
El CESID: Historia de un intento de modernización...

Roldán, en el que se declaró que el objeto material del delito de malversación de caudales públicos «son los caudales públicos y tienen tal consideración los gastos reservados, ya que se configuran, tanto con anterioridad a la Ley 11/95, como con posterioridad a la misma, como unos créditos presupuestarios destinados a aquellos gastos necesarios, para la defensa y la seguridad del Estado y caracterizados por la prohibición de publicidad y por su especial sistema de justificación y control» ${ }^{109}$. También se planteó el problema respecto al caso, recientemente sentenciado sobre fondos reservados en el que estaban imputados los ex-ministros de Interior Barrionuevo y Corcuera y el ex Secretario de Estado de Interior Vera, entre otros.

Sin embargo, el momento más apasionado del debate se articuló en torno a la posibilidad de que los tribunales pudieran controlar la clasificación de documentos. El problema del control judicial de la clasificación de documentos se suscitó por primera vez cuando el Juzgado de Instrucción $n^{\circ} 5$ de la Audiencia Nacional (del que es titular Baltasar Garzón) dictó el 11 de octubre de 1995, requiriendo al Ministerio de Defensa para que le entregara determinados documentos que se hallaban clasificados como secretos. Ante la negativa del Ministerio de Defensa, el Juez entabló conflicto de jurisdicción contra el Ministerio que fue resuelto por la Sala Especial de conflictos prevista en el art. 38 LOPJ $^{110}$. Aquí debemos dejar al margen las cuestiones formales acerca de si en el caso concreto la solución idónea era el recurso al Tribunal de Conflictos Jurisdiccionales ${ }^{111}$ y de si el Tribunal en su sentencia no actuó ultra vires asumiendo funciones jurisdiccionales de control que no le correspondían ${ }^{112}$. Por lo que afecta al tema aquí desarrollado, la resolución del caso por el Tribu-

109 SAP Madrid , sec. $6^{\text {a }}$, de 24 de febrero de 1998 (ponente: Rodríguez González), FJ $4^{\circ} ;$ STS $\left(2^{a}\right)$, de 21 de diciembre de 1999, (ponente: Conde-Pumpido).

110 Sentencia del Tribunal de Conflictos de Jurisdicción, de 14 de diciembre de 1995 (ponente: Esteban Álamo).

111 Se ha argumentado que este órgano, inspirado en el modelo francés de justicia, no resulta adecuado para el nuestro porque, a diferencia de Francia (donde la jurisdicción es compartida por tribunales y administración), en España toda la jurisdicción corresponde a los tribunales (LOZANO, "El sistema de conflictos...», cit., p. 440-441).

112 LOZANO, «El sistema de conflictos...», cit., p. 448-449; MoRAles GarcíA, "Fondos reservados...», cit., p. 444; en contra y defendiendo que el Tribunal ejerció correctamente su jurisdicción, Luis María DíEz-PicAzo, «El secreto de Estado en el proceso penal: a propósito de la sentencia del Tribunal de Conflictos Jurisdiccionales de 14 de diciembre de 1995», en Sobre sécretos oficiales, Madrid, Civitas, 1998 (y antes en La Ley de 15 de enero de 1996), p. 24-25. 
nal de Conflictos se articuló a partir de sólidas premisas ${ }^{113}$. Frente a la sentencia del Tribunal de Conflictos de Jurisdicción se interpuso recurso de amparo ante el Tribunal Constitucional por la acción popular (que no fue, obviamente, parte en el conflicto jurisdiccional) personada en el asunto que investigaba el juez de instrucción. En el recurso se alegó la vulneración de los derechos a la tutela judicial, a la igualdad de armas en el proceso y al juez ordinario predeterminado por la ley. El TC inadmitió el recurso argumentando que la sentencia del Tribunal de Conflictos dispone el modo y forma en que el órgano judicial ha de proceder para interesar del Consejo de Ministros aquella información clasificada como secreta que pueda ser de interés para la instrucción penal «y a esto se limita la parte dispositiva de la Sentencia que, en puridad, se contrae a resolver un conflicto entre órganos y no -como arguye la acusación popular- a declarar que las denominadas materias reservadas no son susceptibles de control jurisdiccional alguno» ${ }^{114}$.

El problema se planteó nuevamente apenas un año después, pero en unos términos ligeramente diferentes que sirvieron para modificar la incipiente doctrina. El nuevo pronunciamiento, producido después de un insólito e inopinado examen in camera de los documentos controvertidos (por supuesto, no previsto por la ley) ${ }^{115}$ despiertó graves interrogantes. En el caso juzgado por el Tribunal de Conflictos, se trataba de un juez de instrucción, que en busca de pruebas inculpatorias, solicitó al Gobierno la desclasificación de secretos de Estado y, ante la negativa del Gobierno, planteó un conflicto de jurisdicción entre el juzgado y el Gobierno. Sin embargo, ahora se trataba de tres casos en los que

\footnotetext{
113 Disentimos así de algún autor que considera que la sentencia es «decepcionante» y de que «los intentos del Tribunal de Conflictos de Jurisdicción de dotar al (...) límite de los poderes judiciales de investigación de una justificación respetuosa de la lógica del Estado de derecho son extremadamente vagos» (DíEz-PiCAZo, "El secreto de Estado...», en Sobre secretos..., cit., p. 27).

114 Providencia del TC, de 20 de mayo de 1996, Sección $1^{\mathrm{a}}$.

115 La medida se acordó por tres autos de 3 de febrero de 1997, confirmados por otros tres autos de 3 marzo de 1997. Mientras alguna autora expresa su sorpresa ante la misma (GARRIDO CUENCA, «El episodio judicial...», cit., p. 235) otro autor alabó el «inequívoco acto de creatividad judicial, pues semejante posibilidad no está contemplada en nuestras leyes procesales», considerando tal prueba como "irreprochable» (Luis María DíEZ-PICAZo, "La desclasificación judicial del secreto de Estado: comentario a las sentencia de la Sala $3^{\text {a }}$ del Tribunal Supremo de 4 de abril de 1997», en Sobre secretos..., cit., p. 51 ss., p. 55-57). Este último autor ya se mostró, antes de las sentencias de 1997, favorable a la introducción de esta técnica mediante un "acto de creatividad judicial» («El secreto...», en Sobre secretos..., cit., p. 35).
} 
distintos jueces de instrucción (el antecitado $n^{\circ} 5$ de la Audiencia Nacional, además del Instrucción $\mathrm{n}^{\circ} 1$ de la Audiencia Nacional, cuyo titular era Gómez de Liaño, y el n ${ }^{\circ} 2$ de San Sebastián), solicitaron al Gobierno la desclasificación de ciertos secretos de Estado. Ahora bien, ante la negativa del Gobierno, lo que se iba a producir no es ya un conflicto de jurisdicción entre los juzgados y el Gobierno (cuyo resultado era previsible que fuera contrario a las pretensiones de los juzgados, de acuerdo con la doctrina ya sentada), sino la interposición de un recurso de amparo por las acusaciones particulares personadas en los tres procedimientos, las cuales invocan el derecho a obtener la tutela efectiva de los jueces y tribunales sin que se produzca indefensión (art. 24.1 $\mathrm{CE}$ ) y el derecho a utilizar los medios de prueba pertinentes para la defensa (art. 24.2 CE). Los tres recursos fueron resueltos en sendas sentencias de la misma fecha a las que se sumaron ciertos votos particulares $^{116}$. Los criterios de solución de los casos por el Tribunal de Conflictos y por el TS fueron diferentes y el TS terminó acordando la desclasificación de dichos documentos.

La conclusión, que prescindía de las estrictas limitaciones que impone el sometimiento al imperio de la ley llegó a ser calificada por Pérez Royo como un "golpe de Estado" y la expresión, aparentemente excesiva, escondía una profunda significación. Con este modo de argumentar, el TS se salió del marco constitucional español, que sigue un modelo europeocontinental en el que los tribunales tienen sus poderes tasados por reglas (sólo pueden hacer lo que la ley les permite hacer), para insertarse en un marco judicial propio del modelo anglosajón, en el que los tribunales no tienen tasadas por reglas legales sus competencias disponiendo de ciertos poderes exorbitantes (injunctions, contempt of court) que les otorgan una posición preeminente. Ahora bien, esa posición y esos enormes poderes, que hacen posible su incursión en el ámbito de las decisiones políticas se ven contrapesados por la posibilidad de sometimiento de los miembros del poder judicial a responsabilidad política (impeachment). La asunción por los tribunales españoles, del modelo continental-europeo, de poderes propios de los tribunales anglosajones, sin el correlativo sometimiento a la responsabilidad (política) a la que éstos últimos se sujetan, era una operación de profunda trascendencia jurídica que sólo puede hacer el poder constituyente.

116 SSTS $\left(3^{a}\right)$, de 4 abril de 1997, en los recursos 602/1996 (ponente: Lescure), 634/1996 (ponente: Cáncer) y 726/1996 (ponente: Trillo). 


\section{Carlos Ruiz Miguel}

150

\section{Conclusión}

La gran paradoja de la regulación general de los servicios de inteligencia es que su funcionamiento debe ser secreto, pero debe estar regulado y garantizado por las instituciones públicas. El éxito de los mismos dependerá siempre de la adecuada realización de este principio. El CESID ha constituido el primer intento español de articular un servicio de inteligencia de acuerdo con estas premisas. No se consiguió plenamente, pero en el intento se adquirió una experiencia valiosa que ya no se podrá olvidar. 